\title{
ARTICLE
}

\section{Performance of the High Pressure Xe detector for the Radiation Monitoring System of the Nuclear Power Plants}

\author{
Byeong Hyeon PARK ${ }^{1}$, Doh Yun JANG ${ }^{1}$, Cheol Ho LEE ${ }^{1}$,Byoung Hwi KANG ${ }^{1}$, Han Soo KIM², Se Hwan PARK ${ }^{2}$, \\ Jang Ho HA${ }^{2}$, Wan No LEE ${ }^{2}$, Geun Sik $\mathrm{Choi}^{2}$, and Yong Kyun KIM ${ }^{1, *}$ \\ ${ }^{I}$ Department of Nuclear Engineering, Hanyang University, Seoul, Korea \\ ${ }^{2}$ Korea Atomic Energy Research Institute, Daedeokdaero, Daejeon, Korea
}

\begin{abstract}
High-pressure Xe (HPXe) ionization chamber is considered as an ideal detector for the Radiation Monitoring System (RMS) in nuclear power plants, since detector response has been shown to be uniform over large temperature ranges up to $170^{\circ} \mathrm{C}$. By using the MCNPX simulation code, the energy spectra were calculated with respect to the thickness of the outer shell and the dependence on the incident direction of the radiation sources in the nuclear power plant. A cylindrical HPXe ionization chamber, which was configured with a shielding mesh to improve its energy resolution, was designed and fabricated. With the gas purification and injection system, Xe and $7 \%{ }^{4} \mathrm{He}$ was purified and injected into the chamber and the performances of the chamber were evaluated. The leakage and saturation currents of the chamber were measured with an electrometer, respectively. Linearity against dose rates was also measured with ${ }^{226} \mathrm{Ra}(0.906 \mathrm{mCi})$ radiation source and the 0.994 of root-mean-square value was estimated. In a future work, energy spectra with the fabricated HPXe ionization chamber will be measured and compared with the simulated energy spectra.
\end{abstract}

\section{KEYWORDS: HPXe detector, radiation monitoring system, ionization chamber}

\section{Introduction}

High-pressure xenon (HPXe) gas is one of the attractive gamma-ray detection gas media due to its physical and nuclear properties. An HPXe chamber has a high detection efficiency for gamma-ray energies on the order of hundreds of $\mathrm{keV}$ due to its large atomic number $(Z=54)$, which translates into a high photoelectric absorption and Compton scattering cross sections. In addition, the Fano factor of HPXe is quite good, measured in the $0.13-0.1$ range. ${ }^{1)}$ These two parameters combine to give HPXe ionization chambers an attractive theoretical energy resolution of about $0.5 \%$ fullwidth half maximum (FWHM) at $662 \mathrm{keV} \gamma$-ray of ${ }^{137} \mathrm{Cs}$ source. For this reason, HPXe ionization chambers are considered as an ideal detector for the radiation monitoring system in nuclear power plants, since this detector response has been shown to be uniform over large temperature ranges up to $170{ }^{\circ} \mathrm{C}^{2-3)}$ The summarized important properties of compressed xenon gas are listed in Table 1, along with a comparison to other common detection media. At present, it is necessary to obtain and to industrialize a radiation detector technology because the utilization of a HPXe ionization chamber is increasing in environmental radiation monitoring systems at domestic nuclear power plants.

*Corresponding Author, Tel No: +82-2-2220-2354, Fax No: +822-2296-2354, E-Mail : ykkim4@hanyang.ac.kr

(C) Atomic Energy Society of Japan
Several designs of HPXe ionization chamber have been developed: parallel-plate, cylindrical, hemispherical chambers and dual-anode configured chambers. ${ }^{4-6)}$ Among a variety of designs, only the cylindrical ionization chambers with the Frisch grid have been used widely and practically and have become commercially available. The reason why cylindrical chambers are commonly used is its sustainment for high operating pressure with a relatively thinner wall thickness and lower operation voltage. Furthermore, the degradation of the energy resolution caused by shielding inefficiency can be minimized. ${ }^{2-3,7)}$

An HPXe ionization chamber can be used as a replacement of a combination of a scintillation detector for detection of radiation energies and a high pressure Ar (HPAr) ionization chamber for environmental dose rate measurement as an alternative environmental radiation detector.

Table 1 The characteristic of HPXe compared with other detecting media

\begin{tabular}{cccc}
\hline Medium & $\begin{array}{c}\text { Density } \\
\left(\mathbf{g} / \mathbf{c m}^{\mathbf{3}}\right)\end{array}$ & $\begin{array}{c}\text { Atomic } \\
\text { number }\end{array}$ & $\begin{array}{c}\mathbf{W} \\
(\mathbf{e V} / \mathbf{i p})\end{array}$ \\
\hline $\mathrm{NaI}: \mathrm{Tl}$ & 3.67 & 11,53 & $\sim 100$ \\
\hline $\mathrm{Ge}$ & 5.33 & 32 & 2.98 \\
\hline $\mathrm{CZT}$ & 6 & $48,30,52$ & 5.0 \\
\hline $\mathrm{HPXe} *$ & 0.5 & 54 & 21.9 \\
\hline$* 50$ atm at a room temperature & &
\end{tabular}

* 50 atm at a room temperature 


\section{Design of the HPXe chamber}

A cylindrical HPXe ionization chamber was designed and fabricated on the basis of the results of the MCNPX simulation code. The parameters considered were the thickness of an outer shell of the chamber and the dependence on the incident direction of the radiation source. The calculated spectrum with ${ }^{137} \mathrm{Cs}(662 \mathrm{keV}, \gamma$-ray $)$ with respect to the thickness of the outer shell are shown in Fig. 1. With the results of the simulation, the HPXe chamber, which was designed so that the chamber can hold the high pressure, minimize the thickness of the wall, as well. The design drawing of the HPXe chamber is shown in Fig. 2.

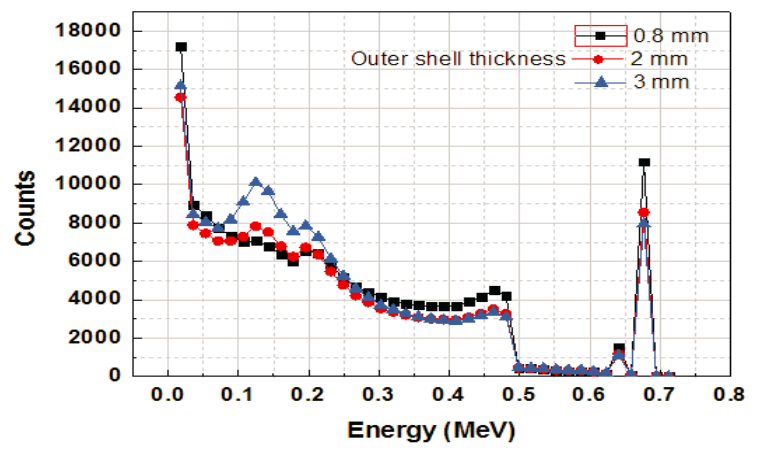

Fig. 1 The simulated $662 \mathrm{keV} \quad \gamma$-ray spectrum with respect to the thickness of outer shell by using a MCNPX code

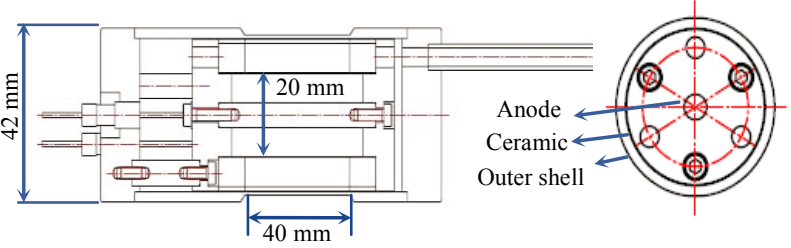

Fig. 2 A design drawing of the HPXe chamber

With the design drawing of the chamber, the dependence of photopeak efficiencies on the incident direction of the radiation source was simulated. The diagram of the simulation and the result come out as shown in Fig. 3. The intrinsic efficiency does not include the solid angle subtended by the detector as an implicit factor and the radiation quanta incident on detector were calculated by $\mathrm{F} 4$ tally in MCNPX simulation code.

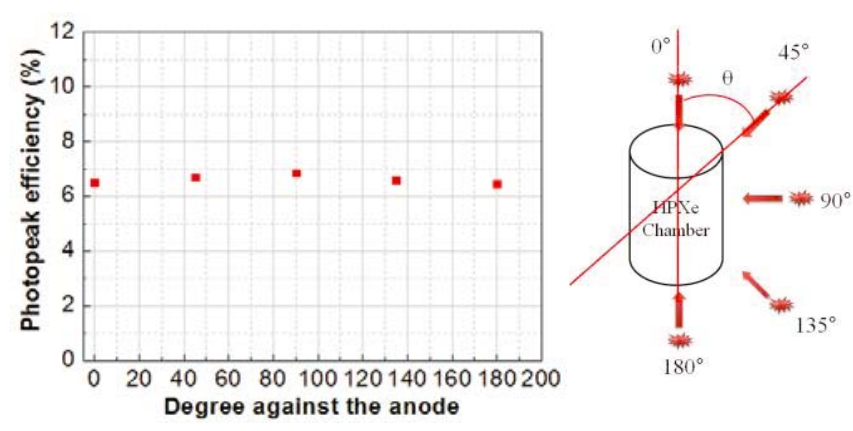

Fig. 3 The efficiencies as a function of the incident direction of the radiation source $\left({ }^{137} \mathrm{Cs}, 662 \mathrm{keV}\right)$
From the Fig. 3, the difference between the maximum and minimum value is only $0.25 \%$, so that its response to the radiation with different incident directions is uniform.

The designed cylindrical ionization chamber was fabricated on the basis of the result from MCNPX code. The diameter and the total length of the ionization chamber are 42 and $130 \mathrm{~mm}$, respectively. The active volume is about 50 $\mathrm{cm}^{3}$. Ceramic was used as the insulators. A shielding mesh, which eliminates the charge contribution of the slow positive ions, was inserted between the collecting electrode and the potential electrode to increase its energy resolution. Three feed-throughs were welded onto the flange of the ionization chamber to connect the electrodes. All the components of the HPXe ionization chamber were washed by DI water and 2propanol several times to get rid of the dust which can cause the sparks in operating. The chamber was baked in vacuum at $100^{\circ} \mathrm{C}$ for 18 hours to eliminate an out-gassing from the chamber materials before a gas filling. The pictures of the inner side of the HPXe chamber and completed one are shown in Fig. 4.
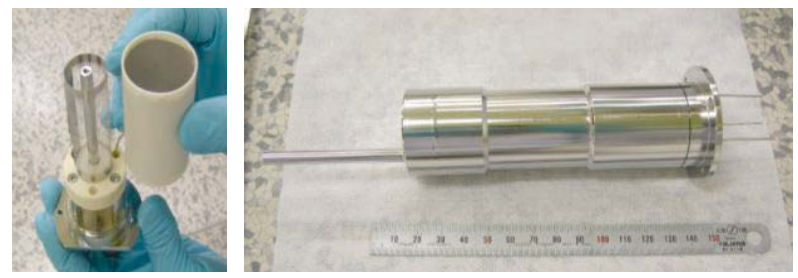

Fig. 4 The inner side of the HPXe chamber and completed one

\section{Performance evaluation of the HPXe chamber}

To evaluate the performance of the fabricated chamber, the Ar gas (99.99\%) was injected at $31.7 \mathrm{~atm}$ and the leakage current was measured by using Keithley 6517A and Caen N471A high-voltage supplier. The leakage currents during the whole experiments were kept in the range of lower than $200 \mathrm{fA}$ and the resultant plateaus were observed.

The Xe gas (99.9995\%) mixed with $7 \%{ }^{4} \mathrm{He}$ was injected into the chamber at a pressure up to 60 atm with a gas purification and injection system. ${ }^{8-9)}$
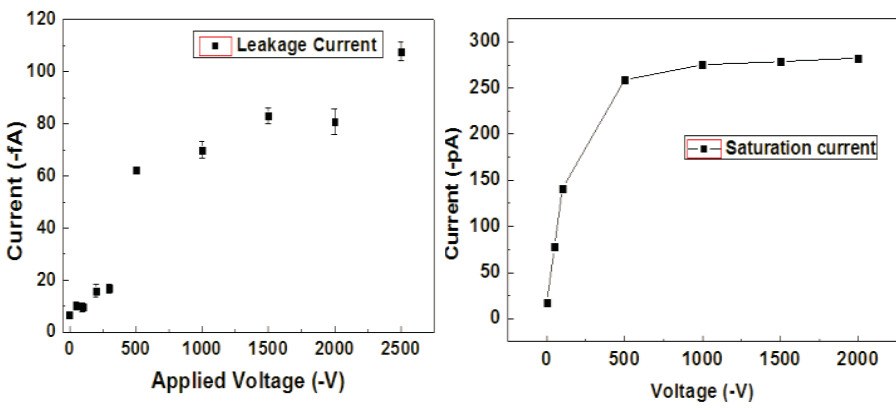

Fig. 5. Leakage currents of the chamber as a function of the biased voltage. The currents were observed in the range of lower than $200 \mathrm{fA}$ (left). The plateaus curve appeared from -500 $\mathrm{V}$ (right). 


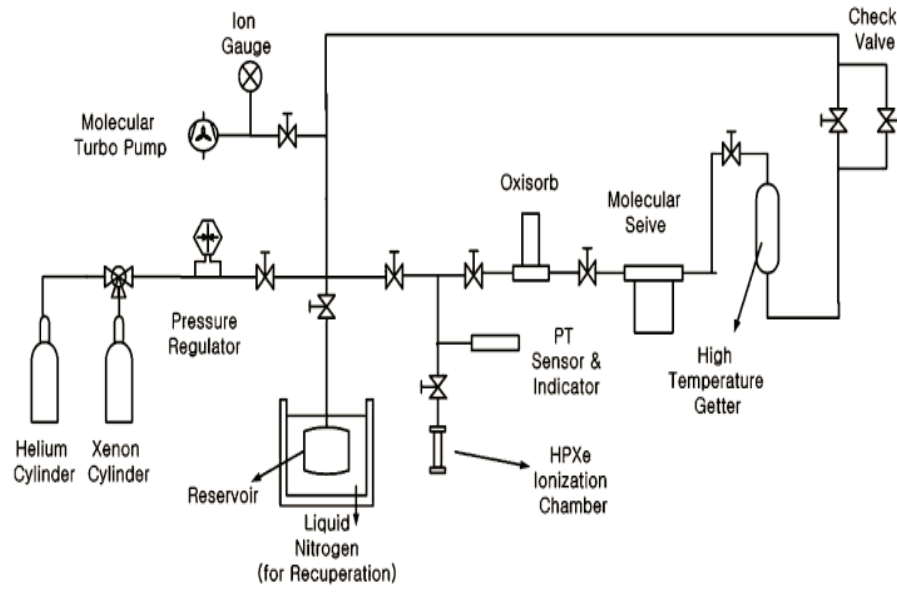

Fig. 6. The gas purification and injection system for the HPXe ionization chamber.

The purification of the $\mathrm{Xe}$ gas is an important parameter in fabrication of HPXe chambers, since electronegative impurities such as $\mathrm{CO}_{2}, \mathrm{O}_{2}$, and $\mathrm{CO}$ can cause degradation in energy resolution. The gas purification and injection system is shown in Fig. 6. As shown in Fig. 8, the reason why Xe gas was mixed with ${ }^{4} \mathrm{He}$ is due to the electron drift velocity in the gas. ${ }^{8)}$

With this $\mathrm{Xe}+7 \%{ }^{4} \mathrm{He}$ filled chamber, linearity test for the performance of the fabricated ionization chambers was performed at a low dose rate. The $0.906 \mathrm{mCi}^{226} \mathrm{Ra}$ source was placed in a low-mass holder at a height of about $100 \mathrm{~cm}$ above the floor and at distances from $40 \mathrm{~cm}$ to $200 \mathrm{~cm}$ from the ionization chamber, which were at the same height on a low-mass stand. A $30-\mathrm{cm}$ thick lead shield with a cross section measuring $10 \times 10 \mathrm{~cm}^{2}$ was interposed on a lowmass stand so as to intercept all the primary rays from the source to all parts of the ionization chambers through a full thickness of the shield. Alignment was checked with a string with a dummy source in place. Slight over-shielding in the

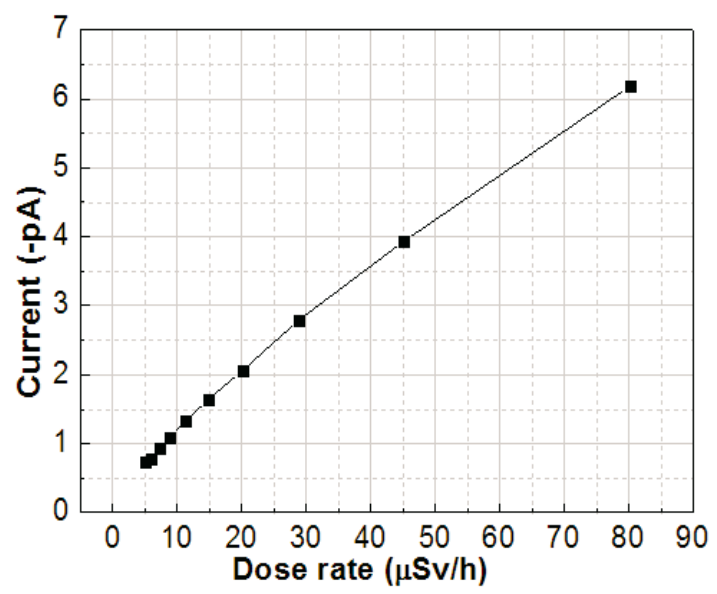

Fig. 7. Linearity of ionization currents from the HPXe ionization chamber as a function of the low dose rates. Root-mean-square linearity was 0.994 . The error bars are smaller than the sizes of symbols.

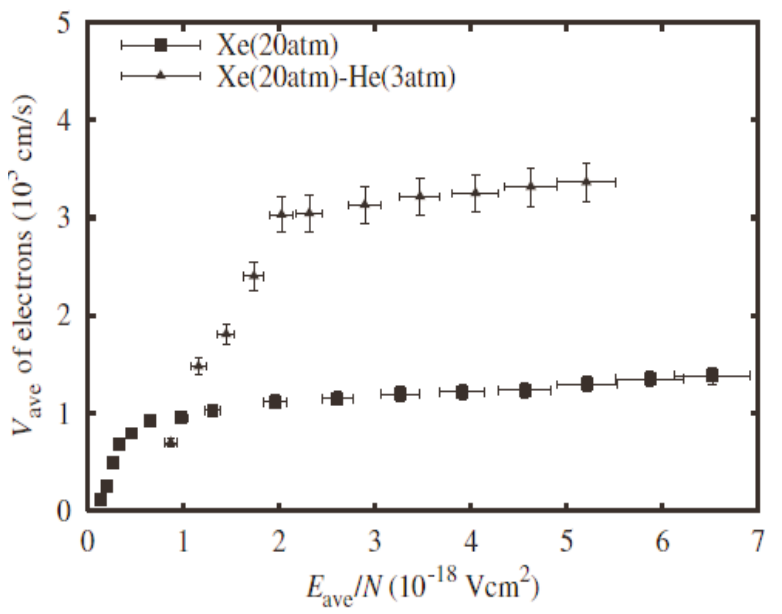

Fig. 8. Averaged electron drift velocities, $V_{\text {ave }}$ for pure $\mathrm{Xe}(20$ $\mathrm{atm})$ and the mixture of $\mathrm{Xe}(20 \mathrm{~atm})-\mathrm{He}(3 \mathrm{~atm})$ marked by squares, $\mathrm{Xe}-\mathrm{He}$ data marked by triangles)

order of $1 \sim 2 \mathrm{~cm}$ was used as this results in a negligible error as opposed to an under-shielding which could produce a significant error. The difference between the average signals was divided by the exposure rate delivered by the source to that at a distance to yield the ${ }^{226} \mathrm{Ra}$ primary beam calibration factor. ${ }^{10)}$

The linearity of the HPXe ionization chamber is shown in Fig.7 and estimated as 0.994 in a root-mean-square value.

\section{Summary}

A cylindrical HPXe ionization chamber, which was equipped with a shielding mesh, was designed on the basis of the results from MCNPX simulation. The dependence on the incident direction of the radiation sources in the nuclear power plant was also simulated and it has a uniform response regardless of the incident direction of the radiation. A gas system was constructed to eliminate the electronegative impurities and to inject the noble gas at up to 60 atm. By using this gas system, $\mathrm{Xe}+7 \%{ }^{4} \mathrm{He}$ up to $60 \mathrm{~atm}$ was injected into the chamber with $0.8 \mathrm{~mm}$ thickness of the outer shell, which means that it can detect the radiation with higher efficiency and less loss of the radiation energy. The tests revealed an outstandingly low leakage currents and 99.4\% linear response against low dose rates. A development of a pressurized gas type radiation detector can be established resulting in the development of a HPXe ionization chamber which can be utilized in high efficient pressurized ionization chamber on condition that the electric noise is reduced. In further study, low noise preamplifier and $\mathrm{RC}$ low filter will be designed and the spectra will be obtained.

\section{Acknowledgement}

This work was supported by the Basic Atomic Energy Research Institute (BAERI, 20090067179), nuclear R\&D program of MEST, Korea and partially supported by the 
Ministry of Knowledge Economy (2008-P-EP-HM-E-060000).

\section{References}

1) Levin, C., et al., "Charge collection and energy resolution studies in compressed xenon gas near its critical point", Nucl. Instrum. Methods A 332, 206-214(1993).

2) Bolontnikov, A.E., et al., "The spectroscopic properties of highpressure xenon", Nucl. Instrum. Methods A 396,360-370(1997).

3) Bolontnikov, A.E., et al., "Improving the energy resolution of high pressure Xe cylindrical ionization chambers", IEEE Trans. Nucl. Sci. 44,1006-1010(1997).

4) Mahler, G., et al., "A portable gamma-ray spectrometer for using compressed xenon" IEEE Trans. Nucl. Sci. 45,1029-1033(1998)

5) Bolontnikov, A.E., et al., "High-pressure Xe detector for gamma-ray astronomy onboard orbital station Mir. In: Proceedings of the International Conference on Liquid Radiation Detectors: Their Fundamental Properties and Applications" (ICLRD) 462-465(1992).
6) Kessick, R., Tepper, G., "A hemispherical high-pressure xenon gamma radiation spectrometer", Nucl. Instrum. Methods A 490, 243-250(2002).

7) Decampo, A., et al., "High pressure argon ionization chamber systems for the measurement of environmental radiation exposure rates", US Atomic Energy Commission, HASL260(1972).

8) S. Kobayashi, V. V. Dmitrenko, T. Doke, V. M. Grachev, N. Hasebe, T. Igarashi, T. Miyachi, H. Okada, E. Shibamura, M. Takenouchi, S. E. Ulin and K. F. Vlasik,. "Measurement of Electron Drift Velocities in the Mixture of Xe and He for a New High-Pressure Xe Gamma-Ray Detector", The Japan Society of applied physics 42, 333-334(2003)

9) V. M. Atrazhev, V. V. Dmitrenko, I. V. Chernysheva, "Electron Transport in Xenon-Hydrogen Gas Mixtures", Technical Physics Letters 30, 301-303(2004)

10) H. S. Kim., et al., "Performance of a high-pressure xenon ionization chamber for environmental radiation monitoring", Radiation Measurements 43,659-663(2008) 